\title{
Bond-valence vector model in analysis of boron coordination sphere
}

Izabela D. Madura $^{1}$, Karolina Kopczyńska ${ }^{1}$, Janusz Zachara ${ }^{1}$

${ }^{1}$ Faculty Of Chemistry Warsaw University Of Technology, Warsaw, Poland

E-mail: izabela@ch.pw.edu.pl

The description of a coordination sphere of a central atom/ion and an understanding of factors influencing its geometry in a given family of compounds is undoubtedly an important issue in many areas of chemistry or biochemistry. Methods grounded on structure correlations are often used to examine this problem provided sufficiently large number of crystal data are available. In these methods a bond-valence vector (BVV) model [1] appears to be a simple tool for identification and quantitative estimation of both steric and electronic factors causing the deformation of the coordination sphere.

In this contribution the systematic analysis of the geometry of three-coordinated boronic acids derivatives with common [CBO2] skeleton will be presented. These compounds are regarded as a significant group taking into account their wide applications in chemistry, biochemistry and material science. They also give a set comprising over 890 [CBO2] fragments thus the analysis with BVV model should be reliable.

In the model, the BVV length is given as $s(i j) *(1-s(i j) / Q(i j))$, where $Q(i j)$ denote the core charge of the central atom and $s(i j)$ is a scalar bond-valence (BV) relating bond-length with bond-valence by two parameters, $r(i j)$ and $b$. These empirical BV parameters in exponential equation [2] for B-O and B-C bonds have been determined recently [3] and they amount to $r(B O)=1.364 A, b(B O)=0.37$ for $B-O$ and $r(B C)=1.569, b(B C)=0.28$ A for $B-C$, respectively. With the calculated $B V V$ lengths the analysis of the distribution of the resultant BVV components allows for a description of both the magnitude and the direction of deformations (see Figure). In- and out-of plane subtle deviations of the "ideal" (sp2) geometry of threecoordinated boron sphere will be discussed and referred to specific modifications of boronic acid. In this context the effect caused by esterification and condensation reactions as well as distortions introduced by intra- and intermolecular hydrogen bonds will be shown. It is worth mentioning that the location of hydrogen atoms on oxygen atoms in syn or anti positions is a key issue in crystal engineering concerning boronic acids. Our contribution will show what kind of strains are introduced while changing from one to another conformation and if the cocrystallization is an effective tool for steering the boronic acid conformation. There will be also a section discussing the out-of-plane distortions, described by vz component of the resultant BVV, where we show that it can be useful for the identification and rough quantification of weak secondary interactions on fourth coordination site of the boron center with the external Lewis base.

[1] Zachara, J. (2007). Inorg. Chem. 46, 9760-9767.

[2] Brown, I. D. \& Altermatt, D. (1985). Acta Cryst. B41, 244-247.

[3] Czerwińska, K. et al. (2016). Acta Cryst. B72, 241-248.

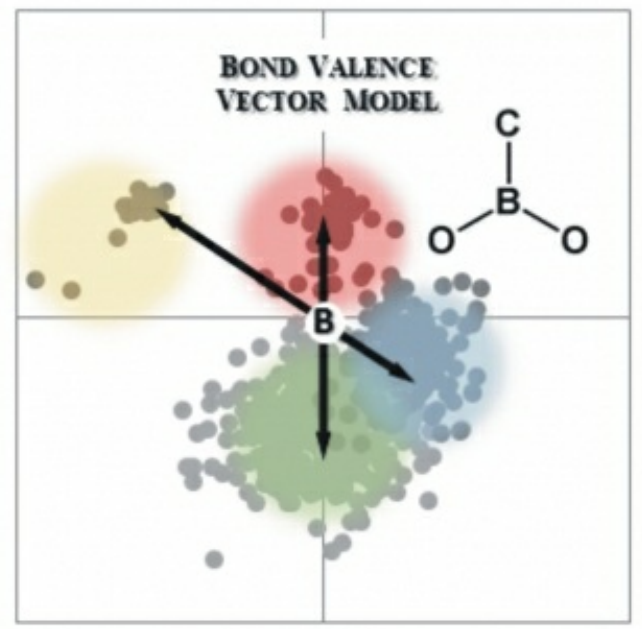

Keywords: bond-valence vector model, boronic acids, geometry of coordination sphere 